\title{
Acute Acalculus Cholecystitis in Dengue Hemorrhagic Fever-A Case Report
}

\author{
MAA MIA, M MOSTAFI, S PERVEEN, NG CHOWDHURY, M A AHMED, SMM RAHMAN
}

\begin{abstract}
Summary:
Presently Dengue appears with its varied features of presentation and progression. We report a case of acalculus cholecystitis in DHF from department of medicine, Combined Military Hospital, Dhaka. The patient presented with fever and diarhea later developing abdominal pain. Her platelet counts were low and Dengue antibody test (Ig M) was positive with altered liver
\end{abstract}

Introduction:

Dengue Fever (DF) is caused by single stranded RNA flavivirus that is transmitted by the bite of female Aedes aegypti mosquito. ${ }^{1}$ Dengue fever is usually a nonspecific and self-limiting biphasic febrile illness but the presentation may range from asymptomatic to Dengue fever, Dengue hemorrhagic fever, Dengue shock syndrome and recently, Expanded dengue syndrome or Isolated organopathy with unusual manifestations. Typical Dengue fever is characterized by high-grade fever, musculoskeletal pain, retrobulbar pain, headache, joint pain, nausea, vomiting and morbilliform rash. Fever, headache and abdominal pain are common manifestations. ${ }^{2,3}$ Atypical presentations like DF complicated by acute acalculous cholecystitis are rare. ${ }^{4}$ Acute acalculous cholecystitis has been described in the course of various diseases and conditions. Occasionally rapid progression to gangrene and gallbladder wall perforation occurs. Therefore, prompt surgical intervention are warranted. Acalculous cholecystitis in the course of dengue is usually a self-limiting disease and surgery if undertaken without proper diagnosis then chances of complication rise as it may be associated with thrombocytopenia, shock and hemorrhage. In these cases, a high clinical suspicion is required to make an early diagnosis and initiate prompt treatment. If

Maj Gen Md Abdul Ali Mia, Brig Gen Mamun Mostafi, Col Shaila Perveen, Col Niamul Gani Chowdhury, Col Mir Azimuddin Ahmed, Lt Col SM Mizanur Rahman

Address for Correspondence: Colonel Shaila Perveen, Classified Medicine spl \& Head of Gastroenterology, CMH, Dhaka. Mob:01819294922

Received: 27 Dec. 2016

Accepted: 8 June 2017 enzymes. Ultrasound showed thick walled gall bladder with clear lumen without stone or sluge, a feature of acute acalculous cholecystitis. The patient was successfully managed conservatively.

Key words and abbreviations: Dengue hemorrhagic fever, Combined military hospital, Acute acalculous cholecystitis.

(J Bangladesh Coll Phys Surg 2017; 35: 155-157)

unrecognized, the delay in treatment may lead to serious complications. This report describes an unusual manifestation of Dengue fever developing acute acalculous cholecystitis.

\section{Case Report:}

A 38 year old female presented with fever and diarrhea with headache, bodyache and severe weakness for 10 days followed by acute onset of abdominal pain and vomiting for 2 days. She was non-diabetic and nonhypertensive but suffering from hypothyroidism for 10 years and getting 50 micrograms of thyroxine daily. On physical examination, she was pale and ill. Her temperature was $39.5^{\circ}$ celcius, pulse $100 /$ minute and blood pressure 100/60 $\mathrm{mm} \mathrm{Hg}$. There was no jaundice or rash on general examination. Abdominal examination revealed diffuse tenderness. Laboratory findings showed hemoglobin level of $10.4 \mathrm{~g} / \mathrm{dl}$ with hematocrit of $32 \%$, TLC was $4.5 \times 10^{9} / 1$ with $87 \%$ neutrophils and $10 \%$ lymphocytes. Platelet count was $70 \times 10^{9} / 1$. Peripheral film showed pancytopenia. Dengue IgM antibodies came out positive. Malarial Parasite was negative and blood culture showed no growth. A clinical diagnosis of Dengue fever with diarrhoea was made. She was admitted and given a standard diet. The patient's condition deteriorated progressively with continued fever, vomiting, increasing abdominal pain and diarrhea with greenish stool in spite of multiple broad spectrum antibiotics. Abdominal tenderness was more marked in right hypochondrium associated with positive Murphy's sign. The ultrasound examination of upper abdomen showed thick walled gallbladder measuring $9 \mathrm{~mm}$ without calculi, mass or sludge in the lumen suggestive of acute acalculous cholecystitis. There was minimal 
free fluid around liver. Serum biochemistry showed total bilirubin of $0.7 \mathrm{mg} / \mathrm{dl}$ with serum alkaline phosphatase of $232 \mathrm{U} / \mathrm{L}$ rising to 920 , serum ALT $289 \mathrm{U} / \mathrm{L}$ and serum amylase $122 \mathrm{U} / \mathrm{L}$. Her creatinine and electrolytes were within normal limits. She was managed conservatively with multiple antibiotics (Meropenem, Linezolid and Metronidazole) and special attention given to her volume status and platelet counts. She was maintained with liquid diet. Her condition stabilized on the 9th day of hospitalization with cessation of fever and regression of right hypochondrial pain. The platelet count rose to $104 \times 10^{9} / \mathrm{L}$ and she could tolerate normal diet without pain. She was discharged on 11th day. After a week, she was fine and only complained of weakness. Abdominal ultrasound showed resolution of the peri-hepatic fluid collection and a decrease in gall bladder wall thickness.

\section{Discussion:}

Now a days clinical features of dengue viral infection have been recognized to take aggressive forms that include atypical diseases like encephalopathy, meningitis, encephalitis, polyneuropathy, Guillain-Barre syndrome, hepatitis including its fulminant form, myocarditis, acalculous cholecystitis, acute pancreatitis, peritonitis, rhabdomyolysis, pulmonary haemorrhage, acute respiratory distress syndrome (ARDS), Macrophage activation syndrome, acute renal injury (AKI), haemolytic-uremic syndrome, disseminated intravascular coagulation (DIC). So some experts have proposed to use Expanded Dengue syndrome / isolated organopathy to incorporate these uncommon clinical symptoms. ${ }^{5}$

In most of the cases Dengue presents with features common to any viral infection. Occasionaly it may even present with features typical of cholecystitis. ${ }^{6}$ Abdominal pain and vomiting are prominent symptoms in dengue and dengue haemorrhagic fever but associated acalculous cholecystitis is an under reported condition. In a series of 131 patients with dengue reported from Taiwan, 10 patients $(7.63 \%)$ had acute acalculous cholecytitis. Two of these patients underwent cholecystectomy and one underwent percutaneous transhepatic gallbladder drainage due to poor resolution. ${ }^{7}$ In another series of 27 dengue patients presenting with fever and abdominal pain in a Northern Indian tertiary care hospital, 17 were found to have acalculus cholecystitis. ${ }^{8}$
Pathophysiology of acute acalculous cholecystitis is bile stasis due to gall bladder dyskinesia and ischemia which results in local inflammation in the gall bladder wall. In severe cases, necrosis of the gall bladder tissue and perforation occurs. In Dengue fever, the main pathophysiological changes are due to increased vascular permeability causing plasma leakage and serous effusion with high protein content that causes thickening of gall bladder wall and gall bladder stasis. Criteria for the diagnosis of acalculous cholecystitis are both clinical and sonographic findings. The clinical manifestations are fever, right upper quadrant pain with tenderness and a positive Murphy's sign. Further evaluation with USG reveal a positive sonographic murphy's sign defined as maximum tenderness at sonographically localized gallbladder, pericholecystic fluid collection and no stone in the gallbladder. The thickness of gallbladder wall in dengue patients exceeds $3 \mathrm{~mm}$ in ultrasonography while thickness exceeding 5 mm indicates a severe course of the disease. ${ }^{9}$ Abdominal sonographic findings in DF are a thickened gallbladder wall, ascites, splenomegaly and pleural effusion. ${ }^{10}$

Contrary to this, some authors have described a typical reticular pattern of gall bladder wall thickness that can be used to diagnose and follow up patients with severe DF but should not be considered as an acalculus cholecystitis. ${ }^{11}$

Acalculous cholecystitis should be suspected in patients infected with dengue complaining of abdominal pain. The sonographic examination in these patients may reveal free fluid around the gallbladder, sonographic Murphy's sign, striations of gallbladder wall indicating its oedema, presence of intraparietal gas and mucosal sloughing. The reported mortality range in acute acalculous cholecystitis accompanying various diseases may reach upto $50 \%$ as compared to $1 \%$ for calculus cholecystitis. Acute acalculous cholecystitis has been described in the course of various diseases and conditions. A study by Gu et al found rapid progression to gangrene and gallbladder wall perforation. ${ }^{12}$ Therefore, strict observation of the patient for prompt surgical intervention are warranted. ${ }^{13}$

Patients in the acute stage of acalculous cholecystitis should receive nothing by mouth. Hydration with intravenous fluids and other supports should be provided. Broad-spectrum antibiotics for enteric and 
biliary pathogen coverage should be administered. The current recommendations include piperacillin/ tazobactam, ampicillin/sulbactam or meropenem (1 gm IV 8h). In severe life-threatening cases, imipenem/ cilastatin. Alternative regimens include a thirdgeneration cephalosporin plus metronidazole (Flagyl, $1 \mathrm{gm}$ IV loading dose followed by $500 \mathrm{mg}$ IV $6 \mathrm{~h}$ ).

\section{Conclusion:}

Acalculous cholecystitis constitutes 10\% (2-15\%) of all acute cholecystitis cases of various aetiology and $40 \%$ of cases are complicated by a gallbladder perforation. ${ }^{14}$ Acalculous cholecystitis in the course of dengue is usually a self-limiting disease and cholecystectomy is usually not indicated in these patients. ${ }^{15}$ If surgery is undertaken without proper diagnosis then chances of complication rise as DF can be associated with thrombocytopenia, shock and hemorrhage. Acalculous cholecystitis should be considered in the differential diagnosis of abdominal pain in patients with dengue and close observation is recommended for avoiding complications.

\section{References:}

1. World Health Organization. Dengue haemorrhagic fever: diagnosis, treatment, prevention and control. 2nd ed. Geneva: WHO, 1997

2. Khan E, Siddiqui J, Shakoor S, Mehraj V, Jamil B, Hasan R. Dengue outbreak in Karachi, Pakistan, 2006: experience at a tertiary care centre. Trans R Soc Trop Med Hyg 2007; 101: 1114-9.

3. Goh BK, Tan SG. Case of dengue virus infection presenting with acute acalculous cholecystitis. J Gastroenterol Hepatol 2006; 21:923-4.

4. Van Troys H, Gras C, Coton T, Deparis X, Tolou H, Durand JP. Imported dengue hemorrhagic fever : description of 1 case presenting with signs of acute alithiastic cholecystitis. Med Trop 2000; (Mar) 60: 278-280.
5. WHO. Comprehensive guidelines for prevention and control of dengue and dengue haemorrhagic fever. Revised and expanded edition. New Delhi: World Health Organization, Regional Office for South-East Asia. 2011.

6. Jaufeerally FR, Surrun SK, Chang PE. Acute acalculous cholecystitis in dengue hemorrhagic fever. Indian J Med Sci 2007; 61:613-4.

7. Keng-Liang Wu, Chi-Sin Changchien, Chung-Mou Kuo, Seng-Kee Chuah et al. Dengue fever with acute acalculous cholecystitis. Am J Trop Med Hyg 2003 Jun; 68(6):657-60.

8. Sharma N, Mahi S, Bhalla A, Singh V. Dengue fever related acalculous cholecystitis in a North Indian tertiary care hospital. J Gastroenterol Hepatol 2006 Apr; 21(4):644-7.

9. Setiawan MW, Samsi TK, Wulur H, Sugianto D, Pool TN. Dengue haemorrhagic fever: ultrasound as an aid to predict the severity of the disease. Pediatr Radiol 1998; 28:1-4.

10. Thulkar S, Sharma S, Srivastava DN, Sharma SK, Berry M, Pandey RM. Sonographic findings in grade III dengue hemorrhagic fever in adults. J Clin Ultrasound 2000; 28: $34-37$.

11. Oliveira GA, Machado RC, Horvat JV, Gomes LE, Guerra LR, Vandesteen L et al. Transient reticular gall bladder wall thickening in severe dengue fever: a reliable sign of plasma leakage. Pediatr Radiol 2010; 40:720-4.

12. Gu MG, Kim TN, Song J, Nam YJ et al. Risk factors and therapeutic outcomes of acute acalculous cholecystitis. Digestion 2014; 90(2):75-80.

13. Shamim M. Frequency, pattern and management of acute abdomen in dengue fever in Karachi, Pakistan. Asian J Surg 2010; 33:107-113.

14. Jhamb R, Kumar A, Ranga GS, Rothi N. Unusual manifestations in Dengue outbreak, 2009, Dehli, India. J Commun Dis 2010; 45: 255-261.

15. Anna Kuna, Agnieszka WroczyDska, MichaB Gajewski, Iwona Felczak et al. A case of acalculous cholecystitis in the course of dengue fever in a traveller returned from Brazil. Int Marit Health 2016; 67(1): 38-4. 